$\mathrm{DTP} / 95 / 42$

May 1995

\title{
On the Coulomb Effects on the W Line Shape at LEP2
}

\author{
V.A. Khoze \\ Department of Physics, University of Durham \\ Durham DH1 3LE, England \\ and \\ W.J. Stirling \\ Departments of Physics and Mathematical Sciences, University of Durham \\ Durham DH1 3LE, England
}

\begin{abstract}
One of the most powerful methods for obtaining a precision measurement of $M_{W}$ at LEP2 is from direct reconstruction of the invariant mass distribution of $W$ bosons produced in $e^{+} e^{-} \rightarrow W^{+} W^{-}$. We investigate the effects on the $W$ line shape, and in particular on the average invariant mass, of phase-space and first-order Coulomb corrections. The latter are shown to have a non-negligible effect on the reconstructed mass, inducing shifts of order $-20 \mathrm{MeV}$ at collision energies above threshold, compared to the Born approximation cross section. The sign and magnitude of the effect can be understood in a simple model calculation in which one of the $W$ bosons is assumed to be stable.
\end{abstract}




\section{Introduction}

A precision measurement of the mass of the $W$ boson, $M_{W}$, is one of the main objectives of the LEP2 physics programme. There has already been considerable progress in the precision determination of $M_{W}$ from $W \rightarrow l \nu$ events at the Tevatron $p \bar{p}$ collider [1], and the level of precision is expected to increase further in the coming years [2]. It is important, therefore, that the potentiality of the $M_{W}$ measurements at LEP2 should be exploited to the full.

An obvious requirement for the success of these precise studies is a high level of reliability of the theoretical predictions for the various experimental observables relating to the different methods for measuring $M_{W}$ from the process $e^{+} e^{-} \rightarrow W^{+} W^{-}$. This in turn requires a detailed understanding of the physical phenomena which describe the production and decay of $W$ bosons at LEP2, in particular of the effects which arise from the large $W$ boson decay width $\Gamma_{W}(\simeq 2.1 \mathrm{GeV})$ [5]. The instability of the $W$ bosons can, in principle, strongly modify the standard 'stable $W$ ' results. For example, an important role can be played by the QED and QCD radiative interferences (both virtual and real) which interconnect the production and decay stages. Particular attention should be paid here to the virtual contributions corresponding to the 'charged' particle poles for which, in contrast to the photon and gluon poles, there is no cancellation from real emissions [6, 7] (see also [8, 9]). The level of suppression of the width-induced radiative effects depends on the 'degree of inclusiveness' of the process. Thus, in the case of the total inclusive cross section for $e^{+} e^{-} \rightarrow W^{+} W^{-} \rightarrow 4 f$, the final-state interaction effects are cancelled up to terms of relative order $\alpha \Gamma_{W} / M_{W}$ or $\alpha_{s}^{2} \Gamma_{W} / M_{W}$. The only exception is the contribution arising from the Coulomb interaction between the slowly moving $W$ bosons [10, 11] (see also [12]). If the $W$ bosons were stable particles, the effect of the Coulomb interaction on the total cross section has been known for a long time [13]. The modifications to the Coulomb corrections which arise from the instability of the $W$ bosons are particularly significant near the $W^{+} W^{-}$production threshold, $\sqrt{s} \approx 2 M_{W}$, but become negligible for collision energies which satisfy $\sqrt{s}-2 M_{W} \gg \Gamma_{W}$. As explained in detail in Ref. [11], the $W$-boson virtuality drastically changes the on-shell value of the Coulomb correction even at $\sqrt{s}-2 M_{W} \gg \Gamma_{W}$, but after integration over the invariant masses of the two $W$ bosons the 'stable- $W$ ' result is restored far above the threshold region.

The electroweak radiative corrections to the $e^{+} e^{-} \rightarrow W^{+} W^{-}$total cross section for the production of stable $W$ bosons are now known with high accuracy 14. The instability effects are well under control throughout the energy range accessible at LEP2 $(\sqrt{s} \lesssim 200 \mathrm{GeV})$. This includes the so-called 'colour-reconnection effects' [15, 16, 17] - non-perturbative hadronization dynamics which may affect the $W^{+} W^{-} \rightarrow 4 q$ decay channel and which have recently attracted much attention.

\footnotetext{
${ }^{1}$ A summary of these methods can be found, for example, in Refs. [3, 价.
} 
Two different methods have been proposed for a precision measurement of $M_{W}$ at LEP2. The first uses the method of direct kinematic reconstruction of $M_{W}$ from the decay channels

$$
\begin{aligned}
& W^{+} W^{-} \rightarrow q \bar{q} q \bar{q}, \\
& W^{+} W^{-} \rightarrow q \bar{q} l \nu .
\end{aligned}
$$

The second method uses the strong dependence of the total $W^{+} W^{-}$cross section on $M_{W}$ near threshold to translate a measurement of $\sigma_{W W}$ close to $\sqrt{s}=2 M_{W}$ into a value of $M_{W}$. Both methods have been, and are currently being, studied in great detail, see for example Refs. [3, 4, 18] and references therein. Although both have their advantages and disadvantages, the direct reconstruction method is currently believed to provide the better precision on $M_{W}$. Since it naturally requires a higher collision energy to maximise the event rate, it does not conflict with the requirements of new particle/physics searches.

However, the direct reconstruction method is not without problems. For example, to construct the two $W^{\prime}$ 's from the $q \bar{q} q \bar{q}$ final state in (1) one must in principle attribute all observed hadrons to the 'correct' parent $W$, a procedure which is certainly affected by relatively unknown QCD interconnection corrections [16]. Since a complete description of these effects is not possible at present, one has to rely on model predictions rather than on exact calculations; for details see Refs. [16, 17]. Fortunately, the contribution of the interconnection effects to the systematic error on $M_{W}$ is not expected to significantly exceed the overall systematic error (not including interconnection effects), which is currently estimated at $O(30-40) \mathrm{MeV}$. It is an open question whether Bose-Einstein effects might induce a further uncertainty in the mass determination [16, 19]. Such problems do not of course arise for the semi-leptonic channel (2), but there the event rate is smaller and an unobserved neutrino is present.

The direct reconstruction method is based on measurements of semi-inclusive characteristics of the final state - $W$ boson momenta, jet-jet invariant masses, opening angle between jets, etc. All such quantities could well be much more sensitive to instability effects than the total cross section. For example, as could be anticipated from Ref. [11] (see also below), the modification of the QED Coulomb interaction could induce a systematic shift in the $W$ mass of $O\left(\alpha \pi \Gamma_{W}\right)$, which is of the same order as the target precision. Clearly such effects should be carefully calculated and taken into account in the extraction of the $W$ mass from measured distributions. Note that since Coulomb forces are responsible for the QED interaction between the separate hadronic or leptonic final states of each $W$, their effects could be regarded as an example of 'QED interconnection effects'. These effects are in fact quite universal and do not depend on the particular $W$ decay channels.

In the threshold region the Coulomb contribution dominates the instability effects, and the Coulomb terms can be uniquely separated from the remaining electroweak 
corrections. At higher energies, the width-induced modifications of the differential distributions caused by other radiative mechanisms (for example, intermediate-final and final-final state radiative interferences [6, 9] ) may become just as important.4 Moreover, it is argued in Ref. [9] that in the relativistic domain a cancellation may take place between the different sources of instability effects and that, as a result, the stable $W^{+} W^{-}$result may be restored. In the extreme relativistic limit, $(1-\beta) \ll 1$, where $\beta$ is the $W$ velocity, such a cancellation appears quite natural.'p However in the intermediate region $\beta<1$, which is relevant for the LEP2 energy range, the situation is less clear in our opinion, and therefore needs further detailed study. Since $\sqrt{s}-M_{W} \ll M_{W}$ at LEP2, the non-relativistic Coulomb formulae are likely to provide a reasonable qualitative guide to the size of the width-induced effects.

In this study we analyse the effect of the $W$ boson instability, as embodied in the first-order Coulomb formulae of Refs. [10, 11], on the invariant mass distribution of the decay products. As far as we are aware, the necessity to take Coulomb-induced distortion effects in the $W$ mass or momentum distribution into account was first pointed out in Ref. [16]. In order to expose the direct effect of this 'QED interconnection', we make several simplifying assumptions in our analysis: (i) the effects of initial state radiation are not included, although it would be straightforward to take them into account using the standard techniques, (ii) we assume that the $W^{+} W^{-}$final state can be fully reconstructed, and (iii) we neglect possible QCD interconnection effects. Note that our results also apply directly to the process $\gamma \gamma \rightarrow W^{+} W^{-}$.

The paper is organized as follows. In Section 2 we present a simple model calculation in which one of the $W$ bosons is assumed to be stable. Above threshold this is expected to provide a reasonable qualitative understanding. Using this model, we study the effect on the invariant mass distribution of the decaying $W$ of phase-space and first-order Coulomb effects. In Section 3 we study numerically the realistic case when both $W$ bosons are off-mass-shell. Predictions for the shift in the average mass with and without Coulomb corrections are presented. Finally, Section 4 contains our conclusions.

\footnotetext{
${ }^{2}$ Recall that in the relativistic region the Coulomb term is neither uniquely defined nor separately gauge invariant.

${ }^{3}$ It has its origins in the conservation of 'charged' currents. Another example of the vanishing of offshell effects at very high energies was discussed in Ref. [20] (see also [21]), where the gluon radiation pattern corresponding to top quark production and decay was discussed. For the most probable kinematic configuration (quasi-collinear $b$ and $t$ ), the width dependence disappears completely when the effects of emission at the production and decay stages are added coherently. The same behaviour is expected for QED radiation off fast-moving $W$ bosons [22].
} 


\section{A model analysis}

To elucidate the physical origin of the distortion of the $W$ decay mass distribution induced by the Coulomb interaction, it is instructive to consider first a simplified model in which one of the $W$ bosons is assumed to be stable and the other has the standard $W \rightarrow f \bar{f}$ decay modes with decay width $\Gamma_{W}$ (see also [9]).

In the non-relativistic region the differential distribution of the invariant masssquared $s_{1}$ of the unstable $W$ can be written as

$$
\frac{d \sigma}{d s_{1}} \approx \rho\left(s_{1}\right) \sigma_{0}\left(s, s_{1}, M_{W}^{2}\right)\left[\left(1+\frac{\alpha}{\pi} \delta_{H}\right)+\frac{\alpha}{2 \beta} \delta_{C}\right]
$$

where $\sigma_{0}\left(s, s_{1}, s_{2}\right)$ is the $e^{+} e^{-} \rightarrow W^{+} W^{-}$off-shell Born cross section 24] at centre-ofmass energy $\sqrt{s}$, and $\rho\left(s_{i}\right)$ is the Breit-Wigner factorf

$$
\rho\left(s_{i}\right)=\frac{1}{\pi} \frac{\sqrt{s_{i}} \Gamma_{W}\left(s_{i}\right)}{\left(s_{i}-M_{W}^{2}\right)^{2}+s_{i} \Gamma_{W}^{2}\left(s_{i}\right)},
$$

with $\Gamma_{W}\left(s_{i}\right)=\sqrt{s_{i}} \Gamma_{W} / M_{W}$. The coefficient $\delta_{H}$ is the 'hard' first-order radiative correction, $(\alpha / 2 \beta) \delta_{C}$ is the first-order Coulomb contribution (see Refs. [10, 11]), and $\beta$ is the velocity of the $W$ bosons in the centre-of-mass frame. In the non-relativistic approximation $\beta=2 p / \sqrt{s}$ where

$$
p^{2} \approx\left(\sqrt{s}-\sqrt{s_{1}}-M_{W}\right) M_{W}
$$

In our model calculation it will be sufficient to work in the non-relativistic approximation. It is then straightforward to show that in this case $\delta_{C}$ is given by the same formulae as in the realistic case of two unstable $W$ bosons [10, 11], with $\Gamma_{W}$ replaced by $\Gamma_{W} / 2$. Thus

$$
\delta_{C}=\pi-2 \arctan \left(\frac{|\kappa|^{2}-p^{2}}{2 p \operatorname{Re}(\kappa)}\right)
$$

with

$$
\kappa=\sqrt{-M_{W}\left(E+\frac{1}{2} i \Gamma_{W}\right)} .
$$

Here $E$ is the non-relativistic energy of the $W$ bosons,

$$
E \approx \sqrt{s}-2 M_{W}
$$

\footnotetext{
${ }^{4}$ In fact precisely this situation applies to the production of a charged Higgs boson with $M_{H} \simeq M_{W}$ in $e^{+} e^{-} \rightarrow Z^{*} \rightarrow W^{ \pm} H^{\mp}$, since in most models $\Gamma_{H} \ll \Gamma_{W}$. The observation of such a process would be a signature of an exotic Higgs sector, see for example Ref. 23.

${ }^{5} \mathrm{We}$ omit here a trivial overall branching ratio factor for the particular $f \bar{f}$ final state under consideration.
} 
Recall that as a direct consequence of the dominance of $S$-wave $W^{+} W^{-}$production at $\beta \ll 1$, 回

$$
\sigma_{0} \simeq \text { const. } \times \beta+O\left(\beta^{3}\right) .
$$

It is worth mentioning that the higher-order terms in the expansion (9) lead to a net negative correction to the leading $\sim \beta$ behaviour, see for example Fig. 1 of Ref. [18], which originates in the high-energy $\mathrm{SU}(2) \times \mathrm{U}(1)$ gauge invariance cancellation.

For $E \gg \Gamma_{W}$ and in the dominant ('peak') region specified by $\left|s_{1}-m_{W}^{2}\right| \lesssim M_{W} \Gamma_{W}$, one finds

$$
\delta_{C} \simeq \pi-2 \arctan \left(\frac{s_{1}-M_{W}^{2}}{M_{W} \Gamma_{W}}\right) .
$$

Eq. (10) reveals the strong dependence of the coefficient $\delta_{C}$ on the $W$ boson virtuality $s_{1}$. This follows from the general nature of the Coulomb forces between unstable heavy particles [11]. Thus in the large invariant mass tail $\left(s_{1}>M_{W}^{2}\right), \delta_{C}$ is strongly suppressed, while in the small invariant mass tail $\left(s_{1}<M_{W}^{2}\right), \delta_{C} \approx 2 \pi$, i.e. twice the first-order on-mass-shell value. After integration over $s_{1}$, the arctan modification of $\delta_{C}$ averages to zero and the stable $W$ result obtains. Note that while for the total $e^{+} e^{-} \rightarrow W^{+} W^{-}$cross section the stable $W$ result for the Coulomb correction is only strongly modified by instability effects in the narrow energy region $E \lesssim \Gamma_{W}$ (i.e. close to threshold), for the invariant mass distribution the arctan modification of $\delta_{C}$ is essentially independent of energy for $E \gtrsim \Gamma_{W}$. However, far above threshold additional energy dependence will appear due to the screening role of the other QED final-state interaction mechanisms.

For purposes of illustration, it is convenient to rewrite the cross section formula (33) for $\Gamma_{W} \ll E \ll M_{W}$ in the dominant $s_{1}$ (peak) region in terms of the dimensionless variable $x$ where

$$
x=\frac{s_{1}-M_{W}^{2}}{M_{W} \Gamma_{W}} \sim O(1) .
$$

The differential cross section in Eq. (3) then becomes

$$
\frac{d \sigma}{d x} \approx \frac{\sigma_{0}}{\pi} \frac{1}{1+x^{2}}\left[\left(1+\frac{\alpha}{\pi} \delta_{H}\right)+\frac{\alpha}{\beta}\left(\frac{\pi}{2}-\arctan x\right)\right],
$$

with

$$
\beta=\sqrt{\frac{E-\frac{1}{2} x \Gamma_{W}}{M_{W}} .}
$$

The invariant mass distribution $d \sigma / d x$ deviates from the Breit-Wigner $\left(1+x^{2}\right)^{-1}$ form, corresponding to an individual $W$ decay, because of (i) the strong dependence of

\footnotetext{
${ }^{6}$ Throughout this paper we assume that the $\nu$-exchange contribution, which dominates the $e^{+} e^{-} \rightarrow W^{+} W^{-}$threshold cross section, is not suppressed by a particular choice of $e^{ \pm}$beam polarizations. The mass distortion effects discussed here are strictly only valid for unpolarized scattering.
} 
the threshold Born cross section $\sigma_{0}$ on the $W$ momentum, and (ii) the characteristic behaviour of the Coulomb term discussed above. In particular, when $\beta \ll 1$, which corresponds to $x \sim 2 E / \Gamma_{W}$, the invariant mass distribution is strongly suppressed by phase space effects, Eq. (9). These lead to a decrease in the average value of the invariant mass, $M=\left\langle\sqrt{s_{1}}\right\rangle$, in the threshold region by

$$
\begin{aligned}
\Delta M_{B} & =O\left(\frac{\Gamma_{W}^{2}}{E}\right) \text { at } E \gg \Gamma_{W}, \\
\Delta M_{B} & =O\left(\Gamma_{W}\right) \text { at }|E| \leq \Gamma_{W}, \\
\Delta M_{B} & =O(|E|) \text { at }-E \gg \Gamma_{W} .
\end{aligned}
$$

With increasing $\beta$ the negative higher-order (in $\beta^{2}$ ) terms in the expansion (9) become more and more important and, as a result, $\Delta M_{B}$ changes sign (see Section 3 below).

The characteristic dependence on the $W$ virtuality of the Coulomb correction for $E \gg \Gamma_{W}$ always causes a decrease in the average mass compared to the Born prediction:

$$
\Delta M_{C}=O\left(\pi \alpha \Gamma_{W}\right) .
$$

Note that this mainly arises from the Coulomb-induced asymmetry in the tails of the distribution, as discussed above. The shift in the actual position of the peak is numerically rather small.

It is important to emphasize the difference between the predictions of Eqs.(6, 10) and the 'on-mass-shell Coulomb' correction, $\alpha \pi /(2 \beta)$. The latter would induce a shift in the $s_{1}$ distribution towards larger values, throughout the threshold region.

Note that there appears to be a range of collider energies around $\sqrt{s} \approx 190 \mathrm{GeV}$ where the phase space and Coulomb induced distortions are of the same order in their effect on the average mass (see Figs. 3 and 4 below). However at this energy, which is of practical importance for LEP2, other mechanisms (e.g. intermediate-final and final-final radiative interference involving the decay products of the two $W$ bosons) have to be taken into account.

Finally, higher-order $\left(O\left(\alpha^{n}\right), n \geq 2\right)$ Coulomb effects could be numerically more important for the invariant mass distribution than for the total cross section. In principle, it is straightforward to take these into account using the general formalism presented in Ref. [11] (see also [25, 26]).

In summary, we have investigated the qualitative effects on the invariant mass distribution of phase-space and Coulomb corrections using a simple model in which only one $W$ boson is off-mass-shell. In the following section, we shall study numerically the more realistic case of both $W$ bosons being off-shell. As we shall see, the conclusions obtained from our model are unchanged by the more complete analysis.

\footnotetext{
${ }^{7}$ The subscript ' $B$ ' denotes a shift in the average mass due to the Born cross section behaviour.
} 


\section{Invariant mass distributions in $e^{+} e^{-} \rightarrow W^{+} W^{-}$: quantitative discussion}

In the realistic case when both $W$ bosons are off-shell, the $W^{+} W^{-}$cross section can be written

$$
\sigma(s)=\int_{0}^{s} d s_{1} \int_{0}^{\left(\sqrt{s}-\sqrt{s_{1}}\right)^{2}} d s_{2} \rho\left(s_{1}\right) \rho\left(s_{2}\right) \sigma_{0}\left(s, s_{1}, s_{2}\right)\left[1+\frac{\alpha}{2 \beta} \delta_{C}\right],
$$

where $\delta_{C}$ is again given by Eq. (6) and $\beta=2 p / \sqrt{s}$, but now with [1]

$$
\begin{aligned}
p^{2} & =\frac{s}{4}\left[1-\frac{2 s\left(s_{1}+s_{2}\right)-\left(s_{1}-s_{2}\right)^{2}}{s^{2}}\right], \\
\kappa & =\sqrt{-M_{W}\left(E+i \Gamma_{W}\right)} \\
E & =\frac{s-4 M_{W}^{2}}{4 M_{W}} .
\end{aligned}
$$

Note that we have omitted the 'hard' radiative corrections and used the 'relativistic' forms for $p^{2}$ and $E$. In what follows we will use Eqs. (16, 17) to study (i) the invariant mass distribution $d \sigma / d s_{1}$ and (ii) the average invariant mass $\bar{M}$ (which has certain practical advantages as an estimator of $M_{W}$ [16]) defined by

$$
\bar{M}=\frac{1}{\sigma(s)} \int_{0}^{s} d s_{1} \int_{0}^{\left(\sqrt{s}-\sqrt{s_{1}}\right)^{2}} d s_{2} \frac{1}{2}\left(\sqrt{s_{1}}+\sqrt{s_{2}}\right) \rho\left(s_{1}\right) \rho\left(s_{2}\right) \sigma_{0}\left(s, s_{1}, s_{2}\right) \quad\left[1+\frac{\alpha}{2 \beta} \delta_{C}\right] .
$$

Figure 1 shows the normalized distribution $1 / \sigma d \sigma / d s_{1}$ (the $W$ 'line shape') as a function of $\sqrt{s_{1}}$, at three different collider energies, $\sqrt{s}=165,175$ and $185 \mathrm{GeV}$. Also shown, for comparison, is the 'pure Breit-Wigner' form $\rho\left(s_{1}\right)$ which corresponds, formally, to the $\sqrt{s} \rightarrow \infty$ limit. As anticipated in the previous section, the phase space effects give a significant distortion to the distribution, especially close to the $W^{+} W^{-}$ threshold. In particular, the distribution is strongly suppressed for $\sqrt{s_{1}} \gtrsim \sqrt{s}-M_{W}$.

The distributions in Fig. 1 include the first-order Coulomb correction $\delta_{C}$. To see the effect of this, we show in Fig. 2 the ratio

$$
f\left(s, s_{1}\right)=\frac{1}{\rho\left(s_{1}\right)} \frac{1}{\sigma} \frac{d \sigma}{d s_{1}}
$$

at $\sqrt{s}=175 \mathrm{GeV}$ with and without the Coulomb correction. Again we confirm the qualitative behaviour obtained in the model analysis of the previous section: the

\footnotetext{
${ }^{8}$ We use $M_{W}=80.41 \mathrm{GeV} / \mathrm{c}^{2}$ [1] and $\Gamma_{W}=2.092 \mathrm{GeV}$ in our numerical calculations. All other parameters coincide with those used in Ref. [18 (see Table 1 therein).
} 
Coulomb contribution enhances (suppresses) the small (large) mass tail. Note, however, that the effect is numerically much less significant at this energy than the distortion due to phase space effects, which forces $f$ to be very small for $\sqrt{s_{1}} \gtrsim 95 \mathrm{GeV}$.

The measurement of the $W$ mass using the diect reconstruction method at LEP2 involves fitting a measured invariant mass distribution, like that of Fig. 1, by a theoretical distribution (in practice implemented in a Monte Carlo program) in which $M_{W}$ is a free parameter. In this way, measured quantities like the position of the peak or the average invariant mass, both of which are crude measures of $M_{W}$, are corrected to the 'true' value. It should be clear from the above discussion that fitting the measured distribution with a theoretical expression which does not include the final-state interaction effects will induce an error in the mass measurement. To quantify this, we focus our attention on the difference between the average $W$ mass $\bar{M}$ (18) defined by the $s_{1}$ distribution and the input mass $M_{W}, \Delta M=\bar{M}-M_{W}$. As in the previous section, $\Delta M_{B}$ denotes the mass shift using the Born (off-mass-shell) cross section and $\Delta M_{C}$ denotes the additional mass shift from including the $O(\alpha)$ Coulomb correction.

Figure 3 shows $\Delta M_{B}$ as a function of the collider energy $\sqrt{s}$. The behaviour can be understood from Fig. 1, and is exactly as anticipated in Section 2. Near and below threshold, there is a strong phase space suppression for masses $\sqrt{s_{1}}>M_{W}$, and so $\Delta M_{B}<0$. Above threshold, the mass difference grows with increasing collider energy as more and more phase space for large invariant masses opens up.

A problem with this calculation of $\Delta M$ is that the integrals over $s_{1}$ and $s_{2}$ receive contribution from arbitrarily small and large invariant masses (subject only to $\sum \sqrt{s_{i}} \leq$ $\sqrt{s}$ ). In practice, events with very large or very small $f \bar{f}$ invariant masses would not be classified as $W$ decay events. In particular, lower cuts on the $\sqrt{s_{i}}$ are required to eliminate non $-W^{+} W^{-}$backgrounds. f $^{-}$To make a more realistic calculation, therefore, we impose an additional cut,

$$
\left|\sqrt{s_{i}}-M_{W}\right| \leq \delta, \quad i=1,2 .
$$

Note that this cutting procedure will to some extent complicate the calculation of the QED and QCD final-state radiative corrections, because of the reduction of the phasespace for final-state emission. The mass shifts $\Delta M_{B}$ for $\delta=30 \mathrm{GeV}$ and $10 \mathrm{GeV}$ are shown as the dashed and dash-dotted curves respectively in Fig. 3. With this additional mass cut there is less dependence on $\sqrt{s}$, since $\Delta M_{B} \rightarrow 0$ as $\delta \rightarrow 0$ at fixed $\sqrt{s}$. Note also that asymptotic values of $\Delta M_{B} \rightarrow 0.46(0.13) \mathrm{GeV}$ are approached for $\delta=30(10) \mathrm{GeV}$, as $\sqrt{s} \rightarrow \infty$.ण

Figure 4 shows the additional mass shift $\Delta M_{C}$ due to the Coulomb correction. Here we see that as long as the mass cut $\delta$ is not particularly tight, the shift for

\footnotetext{
${ }^{9}$ The actual cut value will in practice depend on the particular final state, collider energy, etc. We choose two illustrative values for our numerical calculations.

${ }^{10}$ This is simply the average value of $\sqrt{s_{1}}$ weighted by $\rho\left(s_{1}\right)$ over the range of integration given in Eq. (20).
} 
$\sqrt{s} \gtrsim 170 \mathrm{GeV}$ is rather constant at $O(-20) \mathrm{MeV}$. This is consistent with the model calculation of Section 2, which predicted a constant negative shift of order $\alpha \pi \Gamma_{W}$ for $E \gg \Gamma_{W}$. As can be derived from Eqs. (16,18), $\Delta M_{C}$ changes sign at lower energies and attains a maximum at threshold $(E=0)$, where the average $W$ momentum is lower, $\langle p\rangle \sim \sqrt{M_{W} \Gamma_{W}}$. The actual maximum value depends on the cut parameter $\delta$. Note once again that the mass shift decreases at fixed $\sqrt{s}$ as the invariant mass cut is tightened, i.e. $\Delta M_{C} \rightarrow 0$ as $\delta \rightarrow 0$.

\section{Conclusions}

The success of the precision measurements of the $W$ boson relies on an accurate theoretical knowledge of the details of the production and decay mechanisms. The favoured 'direct reconstruction' method of measuring $M_{W}$ at LEP2 using the hadronic $(q q q q)$ channel has an important caveat - the colour reconnection effects induced by the strong final-state interaction may obscure the separate identities of the $W$ bosons and thus distort the mass determination [16]. At the moment, these effects are not completely curable theoretically because of the lack of deep understanding of non-perturbative QCD dynamics.

However, there are other effects - originating in purely QED radiative phenomena - which, in principle, prevent the final state being treated as two separate $W$ decays. In this paper we have studied one example of this, the Coulomb interaction between two unstable $W$ bosons which induces non-factorizable corrections to the final-state mass distributions. Of course there is no reason why all such effects cannot, in principle, be computed to arbitrary accuracy in QED perturbation theory, and taken into account in the mass determination. In this paper we have demonstrated explicitly that their emission could lead to a $O(20 \mathrm{MeV})$ shift in the measured mass. $\square$ This shift can only be reduced by imposing a rather tight invariant mass cut, which selects only those events near the peak if the distribution where the distortion is minimized. In particular, we have investigated the effect on the mass distribution of the QED interconnection effects generated by the first-order Coulomb corrections in the threshold region at LEP2. At the highest LEP2 energies, it is likely that we are overestimating the mass distortion effect (see for example Ref. [9]). In this region, therefore, our results should be regarded as only a starting point for futher, more detailed studies. Particularly important in this respect are the QED interactions involving the decay products of the two $W$ bosons, which become essential in the relativistic region.

Finally, we note that similar non-factorizable QED final-state interaction effects could also be important in precision $M_{W}$ measurements at the Tevatron $p \bar{p}$ collider,

\footnotetext{
${ }^{11}$ It is also worth mentioning that a similar effect to that described in this study could also be induced by final-state 'new physics' interactions, for example the exchange between the two $W$ bosons of a new light scalar with a sufficiently large coupling.
} 
for example in the process $q g \rightarrow W(\rightarrow l \nu)+q$. The distortion would then be manifest, for example, in the transverse momentum distribution of the final-state lepton.

\section{Acknowledgements}

We are grateful to the UK PPARC for support. Useful discussions with Victor Fadin and Torbjörn Sjöstrand are acknowledged. This work was supported in part by the U.S. Department of Energy, under grant DE-FG02-91ER40685 and by the EU Programme "Human Capital and Mobility", Network "Physics at High Energy Colliders", contract CHRX-CT93-0319 (DG 12 COMA).

\section{References}

[1] CDF collaboration: F. Abe et al., preprints FERMILAB-PUB-95/033-E, FERMILAB-PUB-95/035-E (1995).

[2] Precision tests of electroweak physics: current status and prospects for the next two decades, Report of the DPF Long-Range Planning Study, Working Group 1: Tests of the Electroweak Theory, convenors: F. Merritt, H. Montogomery, A. Sirlin and M. Swartz, September 1994.

[3] Measurement of the W mass at LEP 200: report from the working group on mass measurement, J. Bijnens et al., Aachen ECFA Workshop (1986).

[4] Report from the Working Group on LEP200 Physics, S. Katsanevas et al., DELPHI 92-166 PHYS250 (1992).

[5] For recent reviews on the physics of heavy unstable particles see: V.A. Khoze, University of Durham preprint DTP/94/114 (1994); R.G. Stuart, University of Michigan preprint UM-TH-95-13 (1995), and references therein.

[6] V.S. Fadin, V.A. Khoze and A.D. Martin, Phys. Rev. D49 (1994) 2247.

[7] V.S. Fadin, V.A. Khoze and A.D. Martin, Phys. Lett. B320 (1994) 141.

[8] K. Melnikov and O. Yakovlev, Phys. Lett. B324 (1994) 217.

[9] K. Melnikov and O. Yakovlev, University of Mainz preprint MZ-TH/95-01 (1995).

[10] V.S. Fadin, V.A. Khoze and A.D. Martin, Phys. Lett. B311 (1993) 311. 
[11] V.S. Fadin, V.A. Khoze, A.D. Martin and A. Chapovsky, University of Durham preprint DTP/94/116 (1994), to be published in Phys. Rev. D.

[12] D. Bardin, W. Beenakker and A. Denner, Phys. Lett. B317 (1993) 213.

[13] A. Sommerfeld, Atombau und Spektrallinien, Bd. 2, Vieweg, Braunschweig (1939); A.D. Sakharov, JETP 18 (1948) 631.

[14] For an up-to-date review see: W. Beenakker and A. Denner, Int. J. Mod. Phys A9 (1994) 4837, and references therein.

[15] G. Gustafson, U. Pettersson and P.M. Zerwas, Phys. Lett. B209 (1988) 90.

[16] T. Sjöstrand and V.A. Khoze, Z. Phys. C62 (1994) 281; Phys. Rev. Lett. 72 (1994) 28.

[17] G. Gustafson and J. Häkkinen, Z. Phys. C64 (1994) 659.

[18] W.J. Stirling, University of Durham preprint DTP/95/24 (1995).

[19] L. Lonnblad and T. Sjöstrand, preprint CERN-TH-95-17 (1995), to be published in Phys. Lett.

[20] Yu.L. Dokshitzer, V.A. Khoze, and S.I. Troyan, in Proc. 6th Int. Conf. on Physics in Collision, ed. M. Derrick, World Scientific, Singapore, 1987, p. 417; University of Lund preprint LU-TP-92-10 (1992).

[21] V.A. Khoze, L.H. Orr and W.J. Stirling, Nucl. Phys. B378 (1992) 413.

[22] Yu.L. Dokshitzer, V.A. Khoze, L.H. Orr and W.J. Stirling, Nucl. Phys. B403 (1993) 65; Phys. Lett. B313 (1993) 171.

[23] A.I. Iogansen, N.G. Uraltsev and V.A. Khoze, JETP Lett. 35 (1982) 153; Sov. J. Nucl. Phys. 36 (1982) 717.

[24] T. Muta, R. Najima and S. Wakaizumi, Mod. Phys. Lett. A1 (1986) 203.

[25] V.S. Fadin and V.A. Khoze, Proc. 24th LNPI Winter School, Vol. I, p. 3, Leningrad (1989).

[26] V.S. Fadin, V.A. Khoze and M.I. Kotsky, Z. Phys. C64 (1994) 45. 


\section{Figure Captions}

[1] The distribution $1 / \sigma d \sigma / d s_{1}$ in $e^{+} e^{-} \rightarrow W^{+} W^{-} \rightarrow 4 f$ production at $\sqrt{s}=165$, 175 and $185 \mathrm{GeV}$. Also shown (dotted line) is the asymptotic form, $\rho\left(s_{1}\right)$, given in Eq. (4).

[2] The ratio of the mass distribution of Fig. 1 to $\rho\left(s_{1}\right)$ at $\sqrt{s}=175 \mathrm{GeV}$, with (solid curve) and without (dashed curve) the first-order Coulomb correction.

[3] The difference between the average mass $\left\langle\sqrt{s_{1}}\right\rangle$ and $M_{W}$, as a function of the collider energy $\sqrt{s}$ (solid curve). Also shown are the mass differences when an additional cut $\left|\sqrt{s_{i}}-M_{W}\right| \leq \delta(\delta=30,10 \mathrm{GeV})$ is imposed.

[4] The additional mass shift from including the first-order Coulomb correction, as a function of the collider energy $\sqrt{s}$ (solid curve). Also shown are the mass differences when an additional cut $\left|\sqrt{s_{i}}-M_{W}\right| \leq \delta(\delta=30,10 \mathrm{GeV})$ is imposed. 


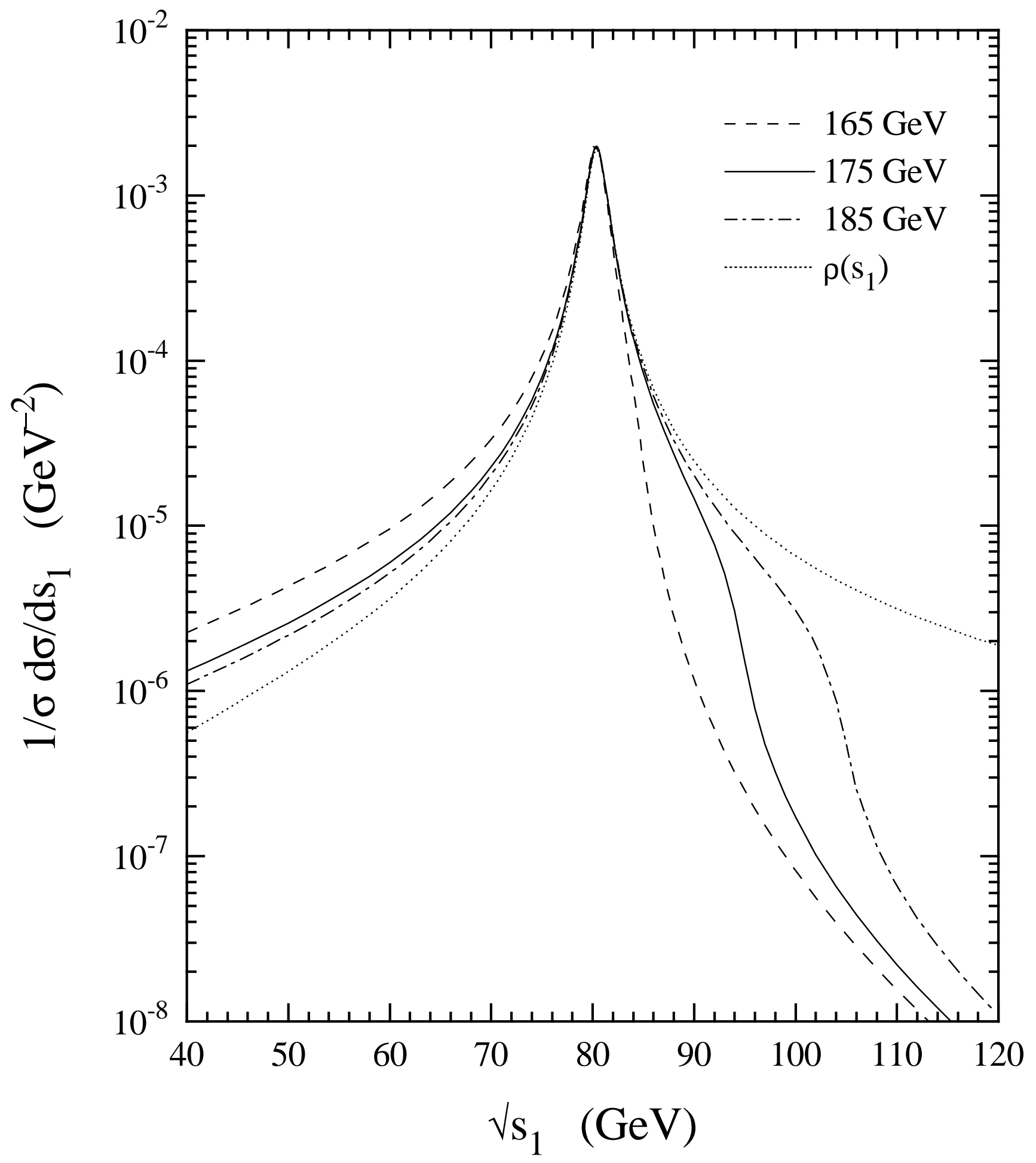

Fig. 1 


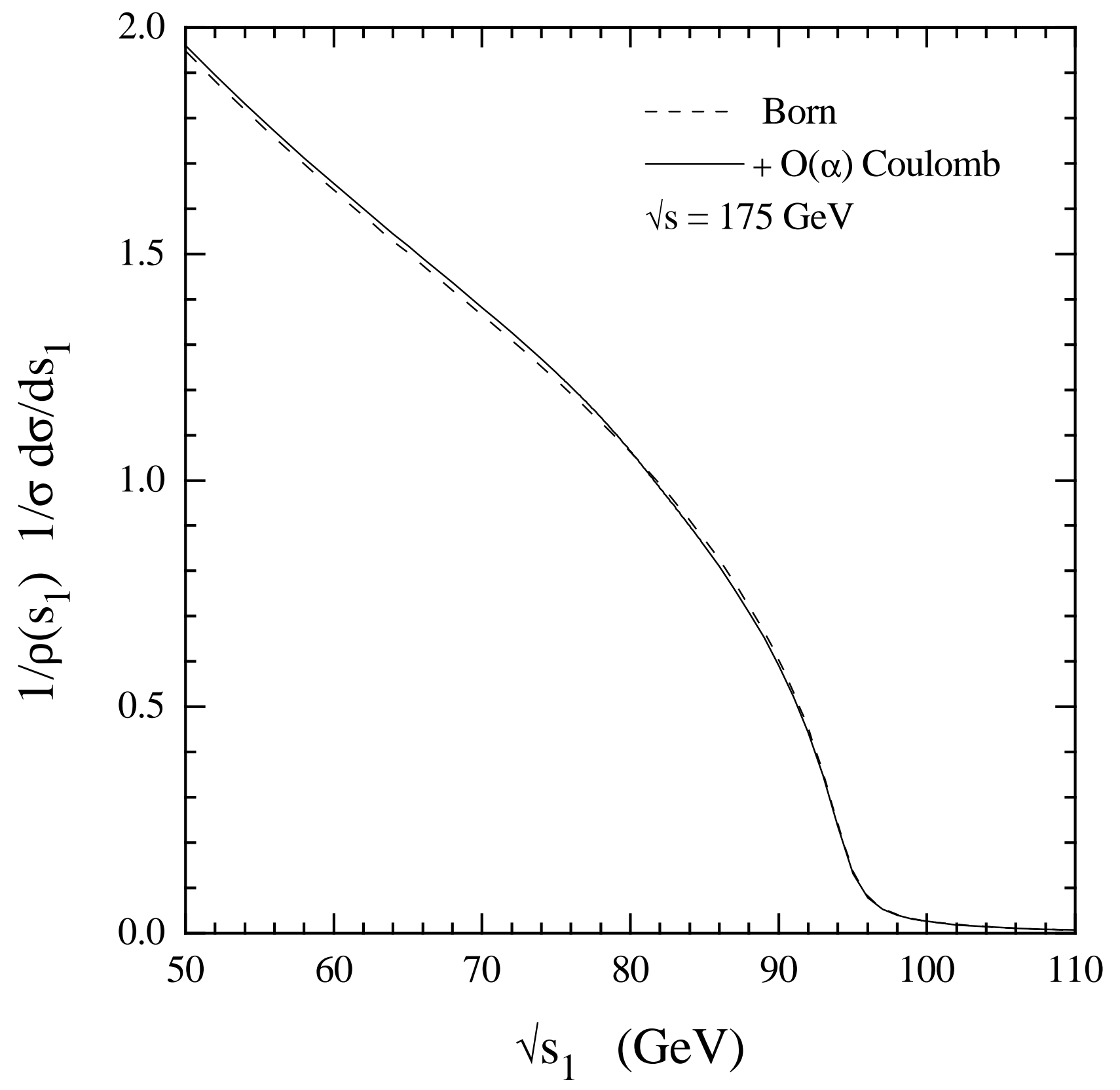

Fig. 2 


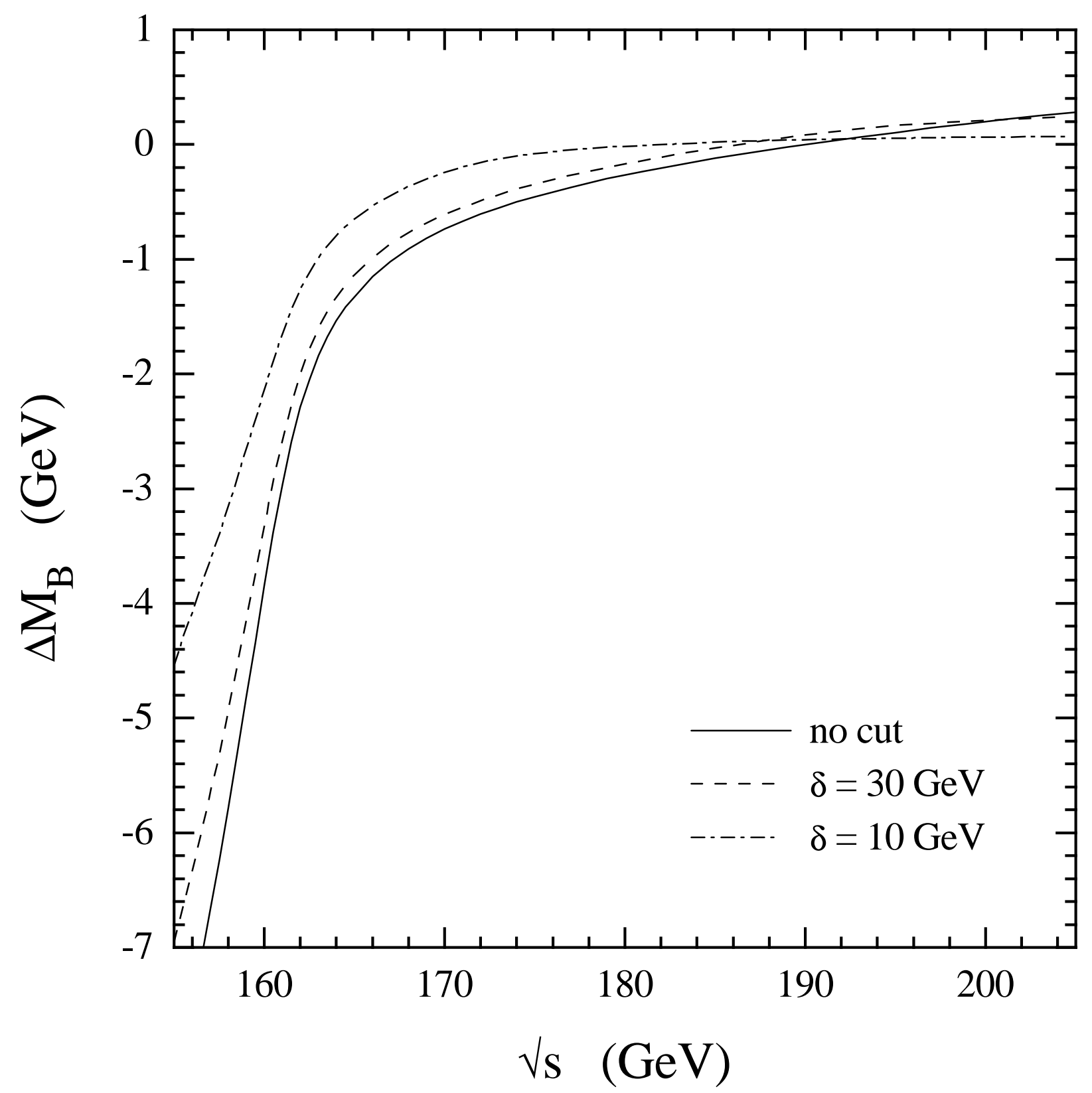

Fig. 3 


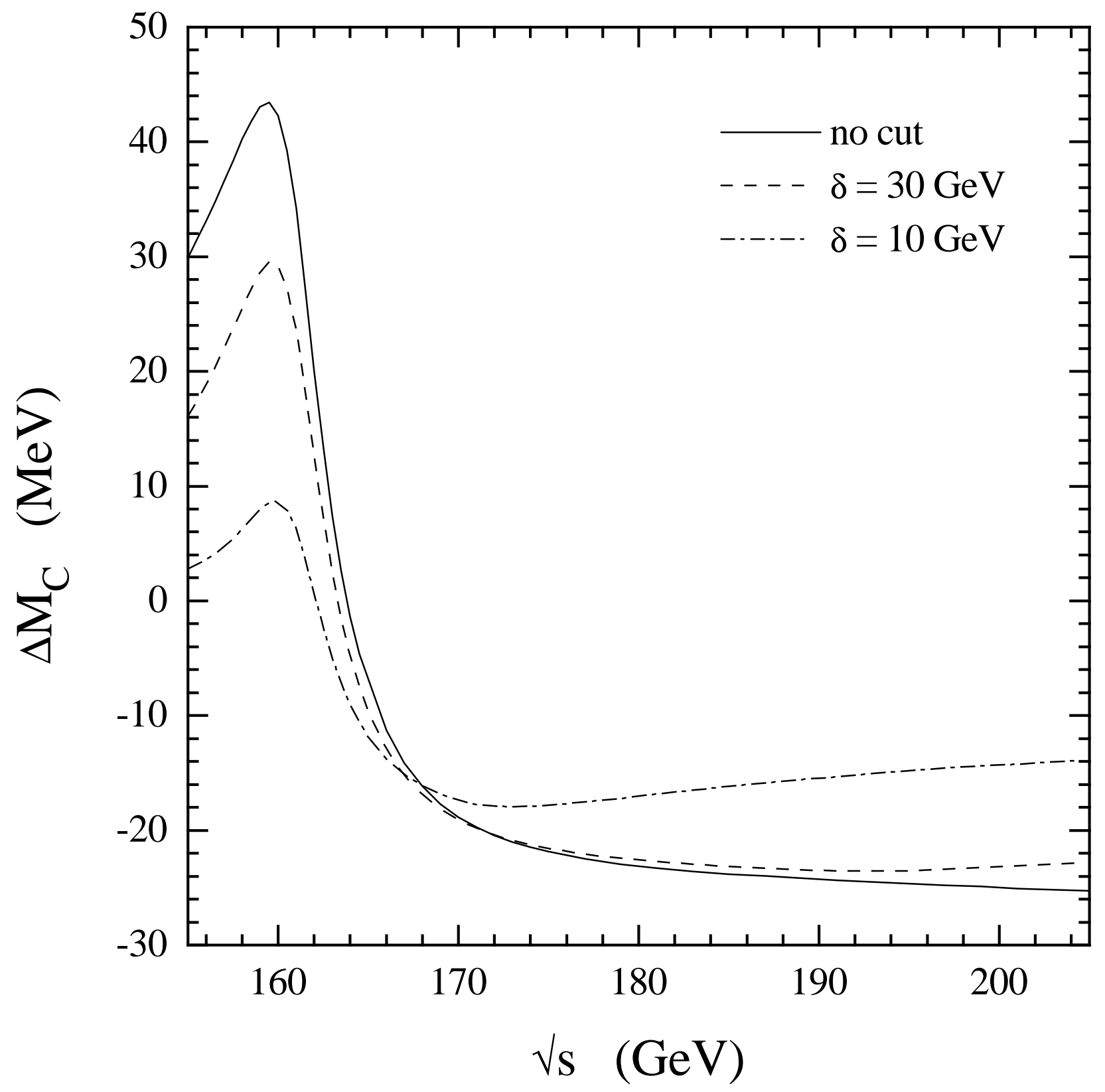

Fig. 4 